cases per 100,000 children aged under 1 year. Sixty-one children met the study case definition $(51 \%$ girls; $41 \%$ born $<37$ weeks gestation; $56 \%$ white, $26 \%$ Asian, $18 \%$ other/unknown ethnicity). Neurodevelopmental abnormalities were reported for $46(75 \%)$ children, including seizures, vision, hearing, and motor abnormalities. The most common identified causes of microcephaly were genetic conditions $(n=5)$ and congenital infections ( $\mathrm{CMV}$, toxoplasmosis, and herpes; $\mathrm{n}=8$ ), however the cause was unknown for $69 \%$ infants. No cases of Congenital Zika Syndrome were identified.

Conclusions The number of children with confirmed severe microcephaly was fewer than estimates based on European congenital anomaly registers. Infants of Asian ethnicity and those born preterm were over-represented. Most children were still under investigation for the cause of the microcephaly. Over three-quarters of children already demonstrated neurological abnormalities; further follow-up will capture later neurodevelopmental outcomes.

\section{G37 PARENTAL NEEDS FOR PSYCHOLOGICAL SUPPORT FOLLOWING A DIAGNOSIS OF PERINATAL STROKE}

${ }^{1} \mathrm{U}$ Khan, ${ }^{2} \mathrm{~T}$ Rapley, ${ }^{3} \mathrm{~A}$ Basu. ${ }^{1}$ Psychology, Newcastle University, Newcastle upon Tyne, UK; ${ }^{2}$ Social Work, Education and Community Wellbeing, Northumbria University, Newcastle upon Tyne, UK, ${ }^{3}$ Neurology, Sir James Spence Institute, Newcastle upon Tyne, UK

\subsection{6/archdischild-2020-rcpch.25}

Background Parents of infants with conditions such as perinatal stroke (PS) often experience feelings of guilt and blame and are at risk of experiencing negative outcomes in terms of psychological wellbeing. This has the potential to adversely affect parent-infant interaction, impacting on the infant. Providing psychological support from an early stage is critically important.

Aims To identify key areas of parental expressed need for support (both met and unmet) following a diagnosis of PS.

Methods Data for the current study was obtained from primary research considering the feasibility of an early parentdelivered therapy intervention (Basu et al. 2018). Eligible participants were recruited from 8 hospitals acting as identification centres. Parents/carers of potential infant participants were identified by clinical staff and their details forwarded to the research team. Secondary analysis of 11 in-depth interviews, exploring parental experiences of delivering the therapy intervention, was carried out using thematic analysis, modelled on the inductive approach by Braun \& Clark (2006).

Results Four overarching themes were identified: 'Lack of information' (due to uncertainty about the future and lack of understanding of clinical terms) was a stressor. 'Need for reassurance' related to wanting information, support, and a sense of purpose. 'Emotional processing' reflects changes in the nature and extent of difficulties experienced in processing the initial stage, coming to terms with the diagnosis and long term adjustment. 'Emotional sensitivity' relates to additional factors related to caring for the infant including social stigma, worrying about developmental discrepancies and the marital relationship, which can act as triggers further distress.

Conclusion The diagnosis of PS led to psychological distress in parents which could be characterised in specific themes. Uncertainty following diagnosis was responsible for significant emotional difficulties. Psychological adjustment to diagnosis was affected by cognitive strategies - but this is a long term and emotionally sensitive process. Findings are discussed in relation to the literature. Recommendations for practice include timely provision of information and resources, restoring self-efficacy, promoting coping strategies to facilitate adjustment, and the compassionate support of healthcare professionals at a time of high vulnerability.

\section{G38(P) KNOWLEDGE, ATTITUDES AND PRACTICE TOWARDS EPILEPSY AMONG PRIMARY SCHOOLS' TEACHERS IN KHARTOUM LOCALITY, SUDAN 2016}

${ }^{1}$ Duaa Omer, ${ }^{2}$ May Abdelruhman. ${ }^{1}$ Faculty of Medicine, University of Khartoum, Khartoum, Sudan; ${ }^{2}$ Department of Community Medicine, University of Khartoum, Khartoum, Sudan

10.1136/archdischild-2020-rcpch.26

Objective This study aims to assess primary schools teachers' knowledge, attitude and practice towards epilepsy.

Methods and Materials This is a cross sectional study that was conducted using structured questionnaire; self administered to 202 teachers selected through multistage-clustered sampling technique, in Khartoum locality, Khartoum state, Sudan.

Knowledge scoring was done by adding (causes variables+ treatment options risk of transmission +symptoms and signs) $=15$ [less than 5 out of $15>>$ poor knowledge. 5-10 out of $15>>$ average knowledge. more than 10 out of $15>>$ good knowledge]. Attitude score answering correctly to 5 different items and scoring was done [less than 2 out of 5 negative attitudes. 2-3 out of 5 neutral attitudes, more than 3 out of 5 positive attitudes]. Practice scoring was done using 7 items [ $>3$ out of $7=$ poor practice, 3-4 out of 7 average practice $<4$ out of 7 good practice].

Result A total of 202 teachers participate in this study. All respondent have heard about epilepsy, (83.2\%) from their nearby community. About (27.7\%) considered evil spirit as a cause for epilepsy and (20.3\%) answered traditional remedy by herbs and sheikhs as best treatment option. (85.6\%) considered epilepsy as non-contagious disease and (67.8\%) considered epilepsy presentation as convulsion only. About (73.7\%) of the participant have an average knowledge regarding initial procedure for seizures management and report removing sharp and maintain patient safety as a major practice.

Total of $(71.8 \%)$ of teachers have an average knowledge about epilepsy, (59.5\%) have average practice toward epileptic and a percentage of $(66.3 \%)$ have a positive attitude toward epilepsy.

Conclusion and Recommendation Teachers had a relatively average knowledge about epilepsy, positive attitudes toward students with epilepsy and their practice toward student during seizure was generally average. Education campaigns of the general public on epilepsy should be encouraged. The need for a well-structured educational and evidence-based intervention for teachers about epilepsy is essential.

\section{G39(P) FEATURES OF PHYSICAL DEVELOPMENT OF CHILDREN WITH CEREBRAL PALSY}

M Balgayeva, Sh Bulekbayeva, Zh Daribayev. National Children's Rehabilitation Center, The Corporate Fund and University Medical Center, Nur-Sultan, Republic of Kazakhstan

10.1136/archdischild-2020-rcpch.27 
Aims The purpose of this work is to study features of physical development in children with cerebral palsy (CP) brought up in the different conditions.

Methods Sixty children with CP participated in the research. Children were divided into 2 groups: the main group consisted of children brought up in children's community, and the comparison group consisted of children brought up in a family. Physical development of children was assessed using the WHO ANTHRO program, at the same time the body mass index (BMI) and indicators of $\mathrm{Z}$-score $\mathrm{BMI}$ concerning age were calculated.

Results When studying physical development of children it is established that at children of the main group average size BMI was $15.47 \pm 2.65$, and the comparison group - 16.21 \pm 2.89 .

Easy insufficiency of nutrition (Z-score from $-2 \sigma$ to- $1 \sigma$ ) was observed at $26.7 \%$ of the examined children of the main group, and at $23.3 \%$ at the comparison group. Moderate nutritional deficiency (Z-score from $-3 \sigma$ to $-2 \sigma$ ) it was diagnosed for $23.3 \%$ of children of the main group and for $16.7 \%$ at the comparison group, while the largest number of children with moderate nutritional deficiency was determined at the age of 3 to 4 years in both groups. Heavy degree of nutritional deficiency (Z-score $<-3 \sigma$ ) in the compared groups was noted equally (6.7\%).

Overweight corresponding to a moderate increase in nutrition (Z-score from $+1 \sigma$ to $+2 \sigma$ ) was detected in $10 \%$ of children in the main group and in the $6.7 \%$ at the comparison group, increased moderate nutrition (Z-score from $+2 \sigma$ to $+3 \sigma$ ) was determined in $3.3 \%$ of children of the main group and the comparison group.

Studying of correlation dependence between indicators of BMI and weight at the birth did not reveal reliable communication between signs in the studied groups, at the same time the correlation coefficient in the main group was $r=0.205$, and in group comparison of $r=-0.146(p>0.05)$.

Conclusion Assessment of physical development of children with use of the international standards allowed to establish existence of disharmonious development in most of the examined children.

\section{G40(P) A CLINICAL SERVICE EVALUATION OF PSYCHOLOGY PROVISION FOR CHILDREN AND YOUNG PEOPLE WITH EPILEPSY, AND THEIR FAMILIES}

${ }^{1} \mathrm{R}$ Govindan, ${ }^{2} \mathrm{R}$ Ransley, 'IJ Scott-Blagrove, ${ }^{2} \mathrm{D}$ Wood. 'Paediatrics, Hillingdon Hospital, London, UK; ${ }^{2}$ Paediatric psychology, Hillingdon Hospital, London, UK

10.1136/archdischild-2020-rcpch.28

This service evaluation aims to ascertain if there is a need for increased psychology provision for children and young people with a diagnosis of epilepsy.

Sixteen parents completed a survey based on the PedsQL Epilepsy module - Parent version (Young Children-Varni, 1988) and the Strength and Difficulties Questionnaire (Goodman,1997) prior to their child's outpatient epilepsy appointment. The child or young person's level, impact and longevity of difficulties, in addition to the family burden were measured. Each question was scored and presented in a pie chart.

Two parents reported that their child and family had previous psychological support which was helpful. $81 \%$ of parents reported that their child had some level of difficulties impacting their emotions, concentration, behaviour and relationships and $75 \%$ reported some level of concern about their child's quality of life in relation to their epilepsy. $37.5 \%$ of parents felt that these difficulties cause their child quite a lot of distress, and in $12.5 \%$, a great deal of distress. $62.5 \%$ of parents report that these difficulties have been present for over a year. $68.8 \%$ of parents said that their child's difficulties caused some level of burden, with $12.5 \%$ report feeling a great deal of burden on their family. $62.5 \%$ of parents stated that it would be very likely that they would engage with psychological support.

The majority of parents who completed the survey felt that epilepsy impacts negatively on their child's emotions, concentration, behaviour, relationships and quality of life and they would consider engaging with psychological support around their child's epilepsy. The findings emphasise that this population has an increased level of need for support around their psychological wellbeing. Moreover, there is a desire for additional psychological support for children and young people with epilepsy, and their families. Recommendations for the service have been made in light of these findings.

\section{G41(P) ABSTRACT WITHDRAWN}

\section{G42(P) EPIDEMIOLOGY AND OUTCOME OF STATUS EPILEPTICUS IN CHILDREN WITH NEW ILAE DEFINITION}

${ }^{1} \mathrm{C}$ Mitchell, ${ }^{2} \mathrm{~L}$ Dickson, ${ }^{2} \mathrm{~A}$ Ramsay, ${ }^{3} \mathrm{P}$ Leonard, ${ }^{3} \mathrm{C}$ Brand, ${ }^{3} \mathrm{~A}$ McLellan, ${ }^{2,3} \mathrm{~J}$ Shetty. ${ }^{1}$ Newcastle Upon Tyne Hospitals NHS Foundation Trust, Newcastle Upon Tyne, UK; ${ }^{2}$ Faculty of Medicine, University of Edinburgh, Edinburgh, UK; ${ }^{3}$ Royal Hospital for Sick Children Edinburgh, NHS Lothian, Edinburgh, UK

\subsection{6/archdischild-2020-rcpch.29}

Background Status Epilepticus (SE) in children carries significant risk of morbidity and mortality. Previous work has predominantly focused on $\mathrm{SE} \geq 30 \mathrm{~min}$ but a new ILAE definition has been produced following evidence that seizures $\geq 5 \mathrm{~min}$ are associated with negative outcomes. This study aims to evaluate the epidemiology and outcome of SE since the introduction of buccal midazolam, change in ILAE definition and increased involvement of specialist epilepsy nurses.

Methods Multiple datasets were combined to identify all children presenting to accident and emergency $(\mathrm{A}+\mathrm{E})$ between 2011-2017 in the region. Data was collated from electronic health records; including patient demographics, clinical characteristics, acute seizure management and outcomes. This data can be used to study long-term outcomes, including educational outcome, through national data linkage systems.

Results There were 665 children admitted with SE who had 1228 seizure episodes during the study period. SE accounted for $0.38 \%$ (95\%CI $0.34-0.42 \%)$ of annual A+E admissions. Yearly prevalence, calculated using mid-year-population estimate, was 0.8 per 1000 children. $57.3 \%$ of patients were male (95\% CI 53.5-61.1\%) and median age was 3.65 years $(\mathrm{IQR}=6.33, \mathrm{Min}=0.0, \mathrm{Max}=20.97)$. There is a small deprivation effect $(p=0.0006)$ which is most prominent at ages $2-3$. The median number of PS for each child was 1, however, $34.1 \%$ of children had recurrent SE and $5.6 \%$ had $\geq 5$ SE. Median seizure duration was $10 \mathrm{~min} .30 .3 \%$ of seizures lasted between 5-29 min. Recurrent seizures and longer duration 